\title{
Jean Dominique Larrey y su reconocimiento en México
}

\author{
Jean Dominique Larrey and his recognition in Mexico
}

Martha E. Rodríguez-Pérez*, Federico Sandoval-Olvera y Horacio Hernández-Salinas

Departamento de Historia y Filosofía de la Medicina, Facultad de Medicina, Universidad Nacional Autónoma de México, Ciudad de México, México

\section{Resumen}

Se analiza la figura de Jean Dominique Larrey, cirujano militar que participó en las guerras napoleónicas. Los objetivos de estudio consisten en destacar sus aportaciones, entre ellas la creación de las ambulancias que permitieron dar una pronta asistencia al herido. Asimismo, señalar el reconocimiento post mortem que Larrey tuvo en México a través de una agrupación académica fundada por el cirujano Francisco Montes de Oca y Saucedo. Los hechos citados muestran el progreso de la cirugía militar, su institucionalización y profesionalización, así como la comunicación y la actualización de saberes en uno y otro continente.

Palabras Clave: Larrey. Cirugía militar. Ambulancias. Sociedades científicas.

\begin{abstract}
The figure of Jean Dominique Larrey, military surgeon who participated in the Napoleonic wars, is analyzed. The objectives of the study are to highlight their contributions, including the creation of ambulances that allowed the injured to be given prompt assistance. Also note the post-mortem recognition that Larrey had in Mexico through an academic group founded by the surgeon Francisco Montes de Oca y Saucedo. The facts cited show the progress of military surgery, its institutionalization and professionalization as well as the communication and updating of knowledge in one and another continent.
\end{abstract}

Key Words: Larrey. Military surgery. Ambulances. Scientific societies.

\section{Introducción}

Jean Dominique Larrey (1766-1842), cuyo nombre está unido a la historia militar de Francia, fue un cirujano que participó en las guerras napoleónicas y pasó a la historia por varios hechos: por brindar asistencia a los heridos en el campo de batalla, por optimizar los métodos quirúrgicos (entre ellos la técnica de la amputación), por sus actividades docentes y fundamentalmente por idear las ambulancias para los heridos de guerra. Todos estos hechos fueron importantes en su momento y valorados no solo en el ámbito médico sanitarista, sino también en el militar, en particular por el emperador francés Napoleón (17691821), quien le otorgó una distinción. Los conocimientos y las destrezas de Larrey traspasaron fronteras, al grado de que muchos años después de su muerte

\section{Correspondencia:}

*Martha E. Rodríguez-Pérez

Brasil 33, Centro Histórico

Del. Cuauhtémoc

Fecha de recepción: 06-11-2018

C.P. 06020 , Ciudad de México, México

E-mail: martha.eugenia.rp@gmail.com

0009-7411/@ 2019 Academia Mexicana de Cirugía. Publicado por Permanyer. Este es un artículo open access bajo la licencia CC BY-NC-ND (http://creativecommons.org/licenses/by-nc-nd/4.0/).
Cir Cir. 2020;88(3):389-394

Contents available at PubMed www.cirugiaycirujanos.com
Fecha de aceptación: 02-07-2019

$24875 / C I R U .190008$ 
fue reconocido en México. El médico militar Francisco Montes de Oca y Saucedo (1837-1885) le rindió homenaje a través de una asociación académica y una publicación editada en la ciudad de México en 1875. El presente texto muestra las aportaciones de Larrey, hombre hábil e inteligente que contribuyó al progreso de la cirugía en escenarios bélicos y su valoración en México.

\section{Datos biográficos}

Jean Dominique Larrey nació en Beaudéan, poblado situado en los Altos Pirineos. Al ahondar en su vida familiar se comprende bien por qué perfiló su vocación hacia la cirugía. Larrey quedó huérfano a los 13 años; su abuelo fue barbero-cirujano en Tarbes, ciudad francesa cercana a su pueblo natal, y su tío Alexis se desempeñó como cirujano del Hospital Saint-Joseph de la Grave en Toulouse, a donde acudió Larrey para aprender el arte de la cirugía y graduarse a los 19 años. Posteriormente trabajó como médico naval, pero pronto renunció para trasladarse a París, donde los cirujanos se unían para defender sus intereses laborales con el fin de alcanzar un estatus similar al de los médicos. Con la creación de la Real Academia de Cirugía en 1731, los cirujanos se independizaron de los barberos, y años después, en 1768, reivindicaban que la cirugía no era un trabajo manual sino una ciencia'. Así, la capital francesa se tornaba en sede del progreso médico quirúrgico, y fue precisamente allí donde Larrey trabajó bajo la dirección de Pierre Joseph Desault, personaje clave en la Academia de Cirugía, hábil cirujano del Hotel Dieu y profesor de figuras que llegarían a ser grandes profesionales de la salud, entre ellos Xavier Bichat, Jean Nicolás Corvisart y el mismo Larrey² (Fig. 1).

Larrey se vio involucrado en la revolución francesa. Inmerso en los movimientos populares de julio de 1789, condujo a más de mil cirujanos y estudiantes que participaban en los levantamientos que llevarían a la toma de La Bastilla el 14 de julio. Poco después se incorporó al Hotel des Invalides para colaborar como asistente del famoso cirujano Raphael Sabatier ${ }^{3}$.

En 1792 se afilió a la Armada Francesa, de manera que participó en un sinnúmero de batallas hasta 1815. Posteriormente se dedicó a la docencia en la escuela médica militar de Val de Gráce en París, como titular de anatomía y medicina operatoria. Más tarde asistió a los soldados discapacitados que ingresaban al Hotel des Invalides, donde en 1830 fue nombrado cirujano en jefe.

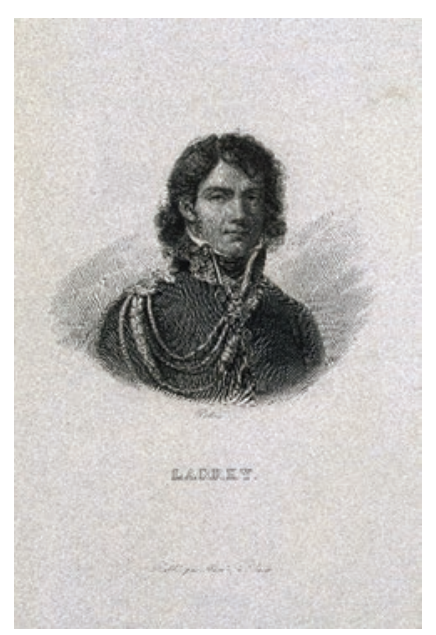

Figura 1. Jean Dominique Larrey, cirujano del ejército napoleónico (Colección Wellcome).

Como cirujano militar, Larrey realizó importantes procedimientos quirúrgicos e ideó las ambulancias de guerra. Falleció en 1842, a los 76 años, en la ciudad de Lyon, a consecuencia de una bronconeumonía. Fue sepultado en Pére Lachaise, el cementerio de la capital francesa que alberga monumentos funerarios de numerosos personajes célebres, entre ellos Frédéric Chopin, Auguste Comte y Jean-François Champollion. Al cabo del tiempo, los deseos de Larrey se cumplieron, ya que en 1992 la Sociedad Francesa de Historia de la Medicina trasladó sus restos a Los Inválidos ${ }^{4}$.

\section{El contexto histórico}

Como cirujano de formación, Larrey vive en una época muy interesante e ilustrativa. Por una parte, la medicina gozaba de progresos significativos: Morgagni sentó las bases científicas del estudio anatomopatológico, cobró importancia la prevención de las enfermedades, se introduce la vacuna contra la viruela por Edward Jenner, Philippe Pinel liberó de las cadenas a los enfermos mentales, Xavier Bichat desarrolló el método anatomoclínico y, entre muchos hechos más, se crearon academias y escuelas prácticas de cirugía que llevaron a la igualdad entre médicos y cirujanos en Francia y otros países europeos.

Larrey presenció el momento en que la cirugía influyó en la medicina interna, cuyo fundamento científico eran los esquemas morfológicos de la patología anatomoclínica ${ }^{2}$, por lo que el especialista se percató de que su quehacer debía apoyarse en la investigación experimental. Sin embargo, el cirujano de finales del siglo XVIII y principios del XIX fue un hombre hábil 
que trabajó bajo las limitantes de la cirugía, el dolor, la hemorragia y la infección, que no serían superadas hasta la segunda mitad de esta última centuria.

Por otra parte, como diría Hipócrates, la guerra es la mejor escuela del cirujano", por lo que se podría decir que Larrey tuvo una excelente formación. Inmerso en un ambiente de angustia y dolor, llegó a percatarse de los múltiples elementos que demandaba la cirugía militar, pues además de la asistencia de heridos y enfermos también contempló su traslado a ciertos puntos de socorro en el mismo frente de batalla, su atención rápida y eficaz, el abastecimiento suficiente de insumos, la capacitación del personal de salud y la adopción de medidas preventivas².

A partir de 1792, cuando Larrey fue nombrado cirujano militar, participó en un sinnúmero de movimientos bélicos. Al estallar la guerra franco-austriaca, Larrey, con el grado de cirujano mayor, formó parte del ejército francés que se instaló en el Rin. Posteriormente se integró a otras campañas contra el Imperio Ruso, el Reino Español, el de Italia y muchos otros. De hecho, en las guerras napoleónicas tomó parte desde la campaña de Egipto hasta Waterloo, que dejaron heridas complejas a causa de los proyectiles.

En 1798, Napoleón otorgó a Larrey el cargo de cirujano en jefe, bajo el que participó en más de 400 combates, en algunos de los cuales fue herido. Este hecho no impidió que Larrey atendiera minuciosamente a sus enfermos y llegara a practicar más de 200 intervenciones, entre amputaciones y desarticulaciones, en un solo día, en la batalla de Borodino, en Rusia ${ }^{2}$. Asimismo, fue nombrado Inspector General del Ejército en $1805^{5}$ (Fig. 2).

Con la derrota de Waterloo, Larrey cayó prisionero bajo el mando de los prusianos y fue condenado a muerte. Cuando estaba a punto de ser fusilado fue reconocido por dos personas, un médico alemán que había asistido a sus lecciones y el hijo de un oficial prusiano herido por las tropas francesas y atendido por el propio Larrey, quienes impidieron su muerte ${ }^{2}$.

\section{Aportaciones de Larrey}

Por su experiencia en el campo de batalla, Larrey señaló que una extremidad muy dañada debía ser amputada inmediatamente. La realización temprana de la amputación sería menos dolorosa y con menor riesgo de hemorragias y de infección. Basándose en Henri Francois Le Dran, Larrey empleó una técnica de amputación con cortes a tres niveles, en piel,

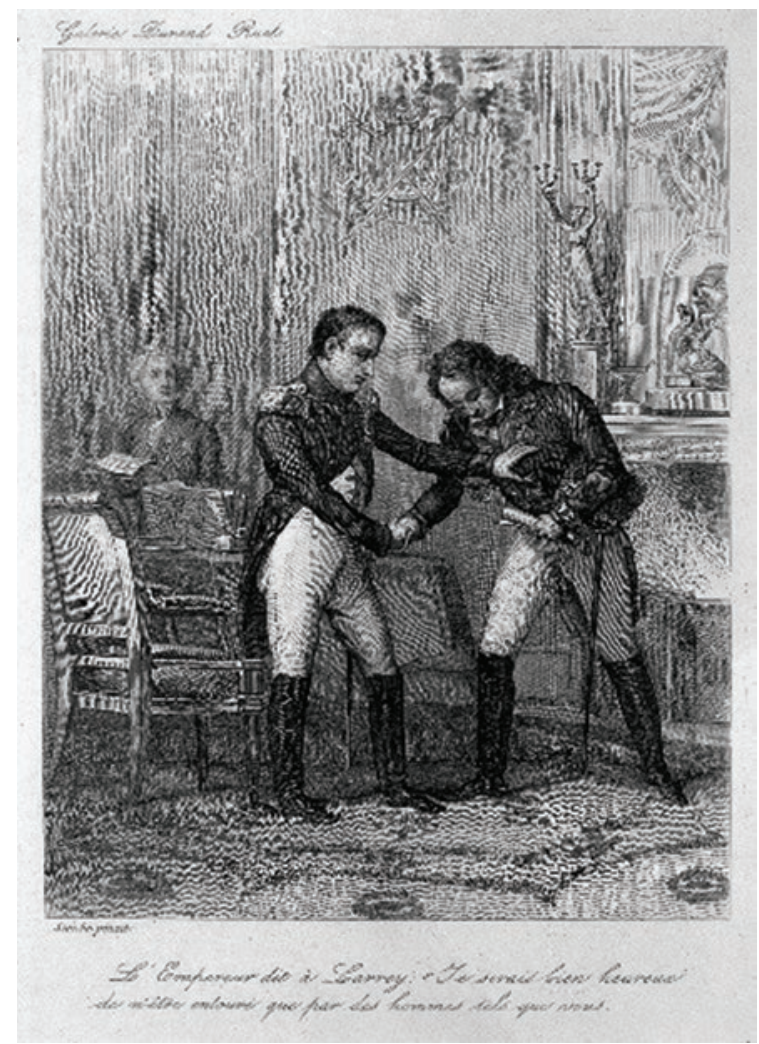

Figura 2. El Emperador Napoleón felicitando al barón Larrey (Colección Wellcome).

músculo y hueso, con buenos resultados, ya que logró una sobrevivencia del $75 \%^{3}$. Le Dran fue un cirujano parisino de la Charité, autor del Traité des opérations de Chirurgie (1731) y de un estudio sobre las heridas por armas de fuego (1737), entre otros textos.

Para prevenir infecciones y gangrenas, Larrey recurrió a la desbridación, eliminando todo tejido necrótico. Para los abscesos acudió al drenaje, y en caso de infección diseminada sugería la flebotomía. Por su parte, para las frecuentes fracturas que atendía Larrey, ideó los vendajes de larga duración que ayudaban a la inmovilización de la extremidad ${ }^{6}$. Asimismo, realizó estudios sobre enfermedades epidémicas y endémicas, como la peste, la fiebre tifoidea, la lepra, el tifo, el carbunco, la fiebre amarilla y el tétanos, entre otras. Como clínico, hizo descripciones del escorbuto, del pie cavo y de infecciones oculares contagiosas, e ideó una técnica de alimentación mediante sonda gástrica ${ }^{7}$. Para los traumatismos craneoencefálicos practicaba las trepanaciones, pero solo en casos de lesiones de la duramadre o fragmento óseo más allá de la tabla interna; de no ser así, era mejor el manejo conservador ${ }^{3}$. En temas de cirugía vascular, Larrey describió los aneurismas luéticos y los 
mecanismos de hemostasia para las lesiones arteriales; también mencionó la formación de circulación colateral y la de fístulas arteriovenosas en las heridas.

En otras cosas, Larrey dio lecciones en diversos lugares y tiempos. Además de las clases que impartía en París, citadas líneas atrás, en la expedición francesa a Egipto (1798-1810), donde el ejército galo creó un hospital militar en Kasr el Aini, Larrey dio un curso de partos a las comadronas egipcias ${ }^{6}$.

Asimismo, nuestro biografiado hizo varias contribuciones a la medicina. Un problema real fueron los heridos de guerra, no solo por la lesión en sí, sino también por su ubicación, por encontrarse en los campos de batalla alejados de los servicios médicos que no podían seguir el avance de las tropas. Para solucionar la situación, Jean Dominique Larrey creó un eficiente medio de transporte que permitía trasladar al herido al puesto de socorro más cercano e irlo asistiendo en el trayecto. Este fue la «ambulance volante", la ambulancia o carruaje rectangular cerrado, con ventilación, compuesto con dos ruedas y tirado por caballos. El vehículo permitía introducir una camilla a fin de que el paciente fuera transportado en posición horizontal. Ante la rapidez con que Larrey organizó las ambulancias, Napoleón lo halagó diciendo: "Larrey, por poco estáis listo antes que yo»". Asimismo, Larrey cambió las fundas de pistola de las sillas de montar por bolsas con instrumentos y vendas $^{9}$ (Figs. 3 y 4).

Además de las aportaciones quirúrgicas de Larrey, también dejó una amplia obra escrita. Sus narraciones o Mémoires de chirurgie militaire (1812-1817) dan cuenta de su vida y trabajo como cirujano de los ejércitos napoleónicos. Hasta antes de la introducción de la anestesia, la cirugía era un acto cruento. Larrey escribe en sus memorias «el caos emocional para los cirujanos militares expuestos a los horrores de la guerra, encarando heridas y lesiones complejas sin esperanza, así como las peticiones que rompían el corazón, de aquellos hombres que sufrían en forma desesperante ${ }^{3}$. Otros escritos de Larrey fueron Mémoire sur l'opthakmie régnante en Egypte (1802), Relation historique de l'expédetion de l'armee d'Orient en Égyte et en Syrie (1803), Dissertation sur les amputations des membres a la suite des coups de feu (1803), Recueil de mémoires de chirurgie (1821), Considération sur la fièvre jaune (1821), Clinique chirurgicale (1829-1836), Mémoire sur le cholera-morbus (1831), Copie d'un mémoire sur le cholera-morbus envoyé à St. Petersbourg en janvier (1831), Mémoire

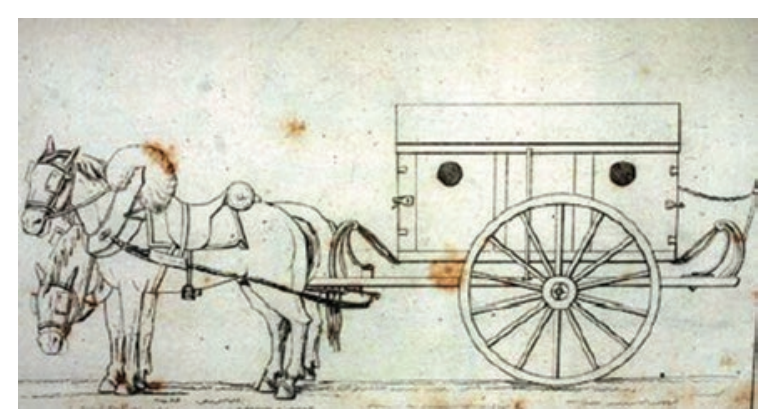

Figura 3. Ambulancia ideada por Larrey (Fresquet, 2005, www. historiadelamedicina.org/larrey.htm.).

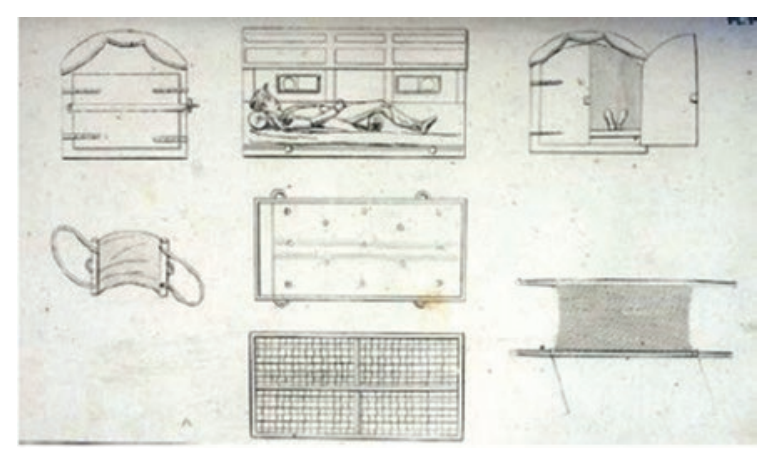

Figura 4. El transporte de heridos en las ambulancias (Fresquet, 2005, www.historiadelamedicina.org/larrey.htm).

sur l'extirpation des glandules salivaires (1841) y $R e$ lation médicale de campagne et voyages de 1815 à $1840(1841)^{10}$.

Larrey fue un cirujano fiel a su ejército, hábil, estratégico, con iniciativa propia y con deseos de aprender e instruir. Estas cualidades fueron reconocidas por Napoleón, quien le otorgó una distinción. Es famoso el siguiente hecho: en una batalla donde las provisiones escaseaban, Larrey sacrificó un caballo para hacer un caldo para uno de sus hombres heridos. Al llegar la noticia al emperador, este ordenó llamar a Larrey y le preguntó "¿Te has atrevido a matar a un caballo de un oficial para alimentar a un herido?". Ante la firme respuesta afirmativa de Larrey, Napoleón le distinguió otorgándole el título de barón ${ }^{5}$. El reconocimiento a Larrey se evidenció nuevamente cuando el emperador dejó escrito en su testamento: «Lego al cirujano en jefe Larrey cien mil francos. Es el hombre más virtuoso que he conocido nunca».

\section{Reconocimiento de Larrey en México}

El 9 de septiembre de 1874, Francisco Montes de Oca y Saucedo fundó en la Ciudad de México la primera agrupación académico-científica constituida por médicos militares en el país, la Asociación Médico-Quirúrgica Larrey, que sesionó quincenalmente en 
el Hospital Militar y contó con un órgano de difusión, los Anales de la Asociación Larrey, en homenaje a nuestro sujeto de estudio, Jean Dominique Larrey. De los Anales se publicaron solo dos tomos, en 1875 y 1876.

Montes de Oca, titulado por la Escuela Nacional de Medicina en 1860, fue catedrático de clínica externa, es decir, clínica quirúrgica. En el marco de la República Restaurada, en diciembre de 1867, el presidente Benito Juárez lo nombró Coronel Subinspector del Cuerpo Médico Militar y Director del Hospital Militar de Instrucción, ubicado en el exconvento de San Lucas. En 1881, Montes de Oca fundó la Escuela Práctica Médico-Militar, antecesora de la Escuela Militar de Medicina. Al año siguiente se le otorgó el grado de general de brigada. Perteneció al cuerpo colegiado más importante de México, la Academia Nacional de Medicina. Dejó escritos en varias publicaciones periódicas, entre ellas la Gaceta Médica de México y los Anales de la Asociación Larrey. Se distinguió por sus habilidades quirúrgicas, en especial por la amputación del tercio superior de la pierna y la desarticulación del hombro, con técnicas un poco diferentes a las planteadas por Larrey ${ }^{11}$ (Fig. 5).

En la primera página de los Anales se lee «La Asociación Larrey se anima en la más noble emulación; tiene por armas el estudio, por tipo a Larrey. Su corazón, es la amistad; su deseo, el progreso de los intereses que representa; su tendencia única, el adelanto médico de México»12. Y para lograr estos fines, los Anales publicaban los trabajos que los socios presentaban y discutían en las sesiones quincenales, así como casos clínicos del Hospital Militar y traducciones de artículos extranjeros. La distribución de la revista fue amplia; cubrió espacios nacionales y extranjeros, entre ellos Francia.

Para rendir un homenaje al cirujano de los ejércitos napoleónicos, Montes de Oca no solo denominó a la asociación recién fundada con el nombre de Larrey, sino que también dio el nombramiento de primer socio honorario extranjero al barón Félix-Hippolyte Larrey, hijo de nuestro sujeto de estudio, quien con fecha de 18 de febrero de 1875 escribió una carta a Montes de Oca agradeciendo tanto la distinción a su padre como la deferencia hacia él. En el mismo documento, Félix-Hippolyte Larrey lamenta las malas relaciones diplomáticas entre México y Francia, y se compromete a enviar al cirujano mexicano una medalla de su padre y la última obra que escribió. El hecho se cumplió cuatro meses después, cuando en un vapor francés que arribó a México llegó Mr. Paul Bergé, portador de

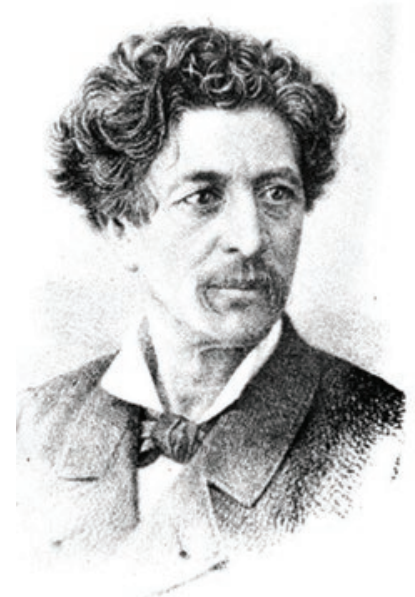

Figura 5. Francisco Montes de Oca, presidente de la Asociación Médica Quirúrgica Larrey.

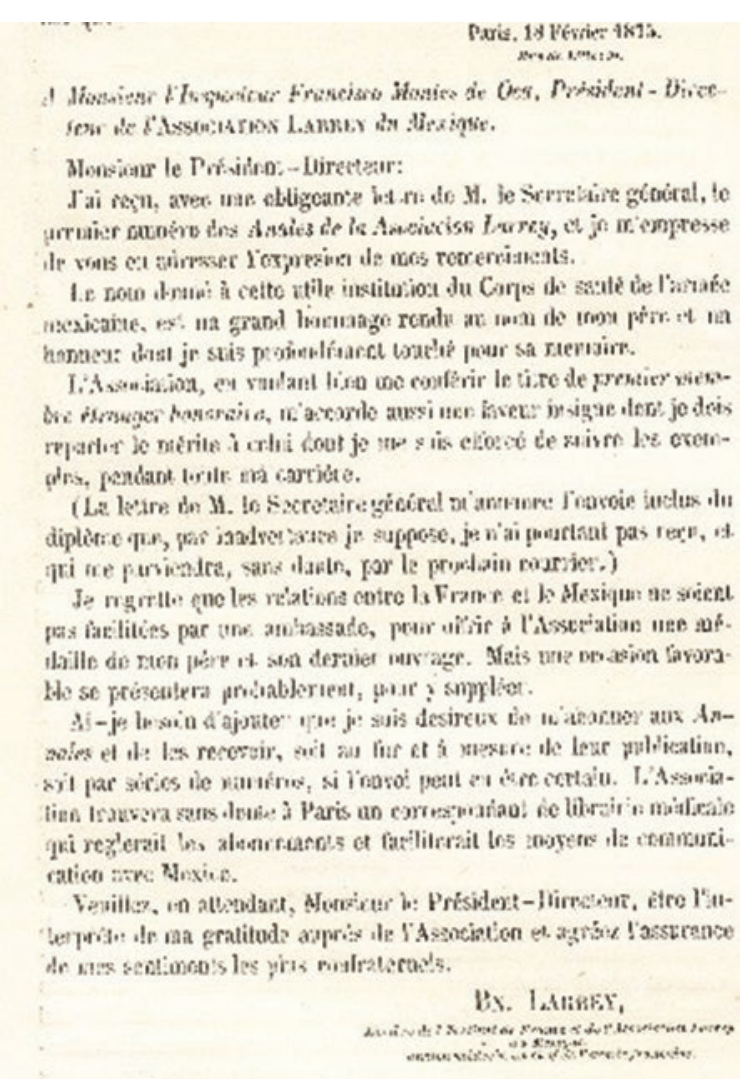

Figura 6. Carta de Félix-Hyppolite Larrey enviada a Francisco Montes de Oca.

una carta que Larrey dirigía a Montes de Oca fechada el 13 de junio (Fig. 6), varias obras de su padre, su retrato y una "preciosa medalla de bronce» que representa por un lado una alegoría en alto relieve llevando abajo MDCCCXXXXVII, y por el frente el busto del ilustre cirujano francés. Al derredor dice «J. D. LARREY NÉ A BAUDÉAN H. (PYRENÉES) LE 8 Jl. 1766 MORT LE 25 Jl. 1842»13. 
El barón Félix-Hippolyte Larrey (1808-1895) también fue médico militar, dedicado a la clínica y a la docencia, y brillante cirujano en el campo de batalla. Entre sus múltiples aportaciones cabe mencionar que incorporó servicios médicos portátiles a los ámbitos bélicos, demostró los beneficios de las fijaciones en las fracturas después de desbridar los tejidos blandos necrosados, y gracias a la época que le tocó vivir, pudo iniciar la aplicación de la anestesia, en particular del éter a partir de 1848, además de utilizar métodos antisépticos con el fin de disminuir las infecciones ${ }^{10}$.

La comunicación entre Félix-Hippolyte Larrey y Montes de Oca se dio en términos totalmente cordiales, dejando al margen los problemas diplomáticos entre Francia y México, que se habían enfatizado con la instalación del imperio de Maximiliano de Habsburgo en territorio mexicano de 1864 a 1867. Como hombres de ciencia, dieron prioridad a la cirugía, que era la especialidad más cultivada en el siglo XIX.

Los hechos citados muestran a Montes de Oca como un gran profesional de la salud interesado en contribuir al progreso de la cirugía mexicana, y no solo a través de la clínica y la docencia, sino también por medio de sociedades académicas, como la que él fundó. La Asociación Médico-Quirúrgica Larrey contribuyó a la profesionalización y la legitimación de la medicina militar. Dedicarla a Jean Dominique Larrey significaba reconocer que la escuela francesa estaba a la vanguardia y que la medicina militar tenía mucho que aportar; por ello, le consagra muchas páginas de este tema en los Anales Larrey.

\section{Comentarios finales}

Ubicados en espacios y tiempos diferentes, se ha estudiado a dos cirujanos militares. El principal, Jean Dominique Larrey, que fue una figura ejemplar, hábil cirujano de guerra que aportó no solo técnicas quirúrgicas propiamente dichas, como la amputación y la desarticulación de extremidades, sino también la pronta asistencia del herido, la valoración del tiempo, para lo cual creó las ambulancias que podían trasladar a los lesionados a puntos de socorro en el mismo campo de batalla. Inmerso en ese ambiente bélico, Larrey antepuso siempre el humanismo que debe caracterizar a los especialistas de la salud, ya que llegó a atender incluso a los heridos del ejército contrario.

El reconocimiento de Larrey se dio en su momento y tras su muerte. La máxima valoración se la otorgó el emperador Napoleón al darle el nombramiento de barón. Tiempo después, y traspasando fronteras, fue distinguido en México por el también hábil cirujano Francisco Montes de Oca, quien encontró el punto exacto en que debían practicarse la amputación del miembro pélvico y la desarticulación del miembro torácico. Montes de Oca evidenció su interés por difundir la ciencia, y de manera especial la cirugía. Participó en la comunidad académica a través de escritos y agrupaciones por medio de las cuales se profesionalizaba su quehacer y este era reconocido como parte de la ciencia moderna. Montes de Oca mostró su admiración hacia la cirugía francesa, hacia la figura de Larrey, que quiso eternizar al dar su nombre a la asociación que fundó. Pero las cosas fueron más allá, pues contactó con el hijo del cirujano del ejército napoleónico, quien mostró su agradecimiento a Montes de Oca de múltiples maneras, difundiéndose todos estos hechos en los Anales Larrey, que constituyeron un «lujoso periódico lleno de interés», como lo consideraba el médico Francisco Flores ${ }^{14}$ y que leía la élite médica en el México del siglo XIX.

\section{Agradecimientos}

Al personal de la Biblioteca de la Facultad de Medicina, de la Universidad Nacional Autónoma de México, por su apoyo para el desarrollo de este trabajo.

\section{Conflicto de intereses}

Los autores declaran no tener conflicto de intereses.

\section{Bibliografía}

1. Porter R. Breve historia de la medicina. De la Antigüedad hasta nuestros días. Trad. I. Cifuentes y T. Carretero. México: Santillana; 2004.

2. López JM, Balaguer E, Ballester R. La cirugía en el Romanticismo. En: Laín P, editor. Historia Universal de la Medicina. Tomo V. Ilustración y romanticismo. Barcelona, España: Salvat; 1978. p. 295-301.

3. De la Garza L. Dominique Jean Larrey. La cirugía militar de la Francia revolucionaria y el Primer Imperio. Cirujano General. 2003;25:359-66.

4. Fresquet JL. Dominique Jean Larrey (1766-1842). Disponible en: http:// www.historiadelamedicina.org/larrey.htm

5. Guthrie DA. History of medicine. London, Great Britain: Thomas Nelson \& Sons; 1946.

6. Guerra F. Historia de la medicina. Madrid, España: Norma-Capitel; 2007.

7. Lyons A, Petrucelli J. Historia de la medicina. Tomo II. Barcelona, España: Doyma; 1982.

8. Manero V. El barón Larrey. Anales de la Asociación Larrey. 1876;:Il:97-9.

9. Cervantes AP, Ruiz JO. Lesiones de guerra en situaciones emergentes de la vida civil. México: Alfil S.A. de C.V.; 2011.

10. Moreno A. Dominique-Jean y Félix-Hippolyte Larrey: el legado de dos cirujanos (padre e hijo). Revista Hispanoamericana de Hernia. 2014;2:23-32.

11. Guarner V. Francisco Montes de Oca y Saucedo, destacado cirujano en el México del siglo XIX. Revista de la Facultad de Medicina de la UNAM. 2010;53:24-9.

12. La Redacción. Anales de la Asociación Larrey. 1875;l:1.

13. Obsequio del Barón Larrey. Anales de la Asociación Larrey. 1875;l:186.

14. Flores F. Historia de la medicina en México desde la época de los indios hasta la presente. Tomo III. Edición facsimilar de 1888. México: Instituto Mexicano del Seguro Social; 1982. 